\title{
Research Article \\ Swirling Flow in a Permeable Tube at Slowly Expanding and Contracting Wall
}

\author{
Sufian Munawar \\ Department of Quantitative Methods, College Business Administration, University of Dammam, Dammam 31441, Saudi Arabia \\ Correspondence should be addressed to Sufian Munawar; sufian.munawar@hotmail.com
}

Received 5 September 2016; Accepted 27 December 2016; Published 19 January 2017

Academic Editor: Sébastien Poncet

Copyright (C) 2017 Sufian Munawar. This is an open access article distributed under the Creative Commons Attribution License, which permits unrestricted use, distribution, and reproduction in any medium, provided the original work is properly cited.

\begin{abstract}
The swirling flow inside a circular elastic tube with expanding and contracting permeable wall in the presence of a uniform magnetic field is studied analytically. The tube is also assumed to be rotating around its axis with an angular velocity. The governing equations for this multidimensional flow are reduced to nonlinear differential equations with similarity transformations. An analytic series solution is obtained by homotopy analysis method (HAM). The effects of physical parameters on various flow characteristics, such as the velocity, skin friction, and pressure variation, have been analysed briefly. The impact of surface expansion/contraction and rotation has been investigated on the internal boundary-layer flow inside the tube of uniform cross-section.
\end{abstract}

\section{Introduction}

The laminar boundary-layer flows inside elastic pipes or tubes have received great attention by numerous researchers since they are widely used in various engineering and biological applications. The examples include the binary gas diffusion, transport of biological liquids through contracting or expanding vessels, pulsating porous diaphragms, urine flow in urethras, natural transpiration and cooling, and the regression of the burning surface in solid rocket motors. Uchida and Aoki [1] initiated the study on the flow inside a tube with deformable wall and one closed end. Ohki [2] discussed the laminar incompressible flow in a semi-infinite porous pipe with expanding and contracting radius in axial direction. A theoretical investigation was performed by Goto and Uchida [3] to study the suction/injection effects in an expanding/contracting pipe. Bujurke et al. [4] investigated the same flow problem by calculating a series solution. Recently, Si et al. [5] calculated the multiple solutions for the contracting or expanding porous pipe at large suction Reynolds number using singular perturbation method. Srinivas et al. [6] obtained analytic series solution for the thermal-diffusion and diffusion-thermo effects in a channel with moveable permeable walls. Currently, the effect of mass transfer and chemical reaction inside a porous tube with expanding or contracting wall was addressed by Srinivas et al.
[7]. The effect of MHD on the same type of flow phenomenon was studied by Srinivas et al. [8]. The flow inside a twisted pipe was investigated by O'Dea and Waters [9] and calculated the solute uptake inside a twisting pipe. Makinde [10] presented a mathematical model describing the flow inside a collapsible tube and obtained an analytic series solution. The effect of variable viscosity and viscous dissipation on the flow in a moving pipe was discussed by Makinde [11]. Xinhui et al. [12] investigated the slip effects in the micropolar fluid inside a porous channel with expanding/contracting walls. Another type of three-dimensional flows in polar coordinates is the boundary-layer flow over cylinder. In this regard, Sprague and Weidman [13] investigated the boundary-layer flow over a twisted cylinder and an asymptotic solution for large range of the Reynolds number was obtained. Keeping this fact in mind the boundary-layer flow over cylinder can also be considered three-dimensional if the surface of pipe moves in two lateral directions. Recently, Munawar et al. [14] made a complete thermodynamical analysis of a three-dimensional viscous flow over a stretching and rotating cylinder. Fang and Yao [15] and Fang et al. [16] discussed other sorts of the threedimensional boundary-layer flows over cylinder which was rotating, expanding, and stretching with respect to its length.

This analysis extends the idea by embarking on the impact of swirling on the flow inside a contracting/expanding semiinfinite porous tube. Since most of the biological fluids are 


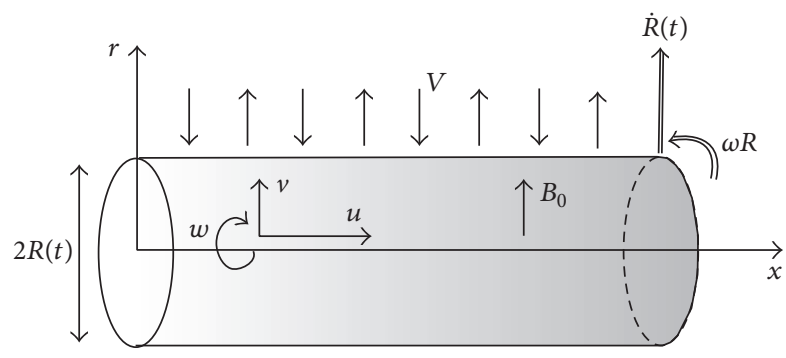

FIGURE 1: Schematic diagram and the coordinate system.

electrically conducting, therefore a uniform magnetic field is taken along the radial direction. An approximate series solution is obtained by using homotopy analysis method (HAM). The convergence and accuracy of the solution are illustrated by plotting convergence tables, plotting the $h$ curves, and calculating the squared residual errors. To validate our HAM solution a comparison has also been made with the results reported by Li et al. [17] using numerical and analytic techniques.

\section{Mathematical Formulation}

Consider an axisymmetric flow of an incompressible viscous fluid inside a semi-infinite horizontally placed expanding and contracting tube. The tube is rotating with a time-dependent angular velocity $\omega(t)$. The radius of tube $R(t)$ is a continuous function of time and the surface of tube is assumed to be uniformly permeable. One end of the tube is kept closed and the wall of the tube expands and contracts with a timedependent rate $\dot{R}(t)$. A uniform magnetic field with intensity $B_{0}$ is applied along the radial direction. Assuming small magnetic Reynolds number, the induced magnetic field is assumed to be negligible. The $x$-axis is measured along the axis of tube and the $r$-axis is perpendicular to the surface (see Figure 1). Under these assumptions the governing equations of motion are given by

$$
\begin{aligned}
& \frac{\partial u}{\partial x}+\frac{1}{r} \frac{\partial(r v)}{\partial r}=0 \\
& \frac{\partial u}{\partial t}+u \frac{\partial u}{\partial x}+v \frac{\partial u}{\partial r} \\
&=-\frac{1}{\rho} \frac{\partial p}{\partial x}+v\left[\frac{\partial^{2} u}{\partial x^{2}}+\frac{1}{r} \frac{\partial}{\partial r}\left(r \frac{\partial u}{\partial r}\right)\right]-\frac{\sigma B_{0}^{2}}{\rho} u \\
& \frac{\partial v}{\partial t}+u \frac{\partial v}{\partial x}+v \frac{\partial v}{\partial r} \\
&=-\frac{1}{\rho} \frac{\partial p}{\partial r}+v\left[\frac{\partial^{2} v}{\partial x^{2}}+\frac{1}{r} \frac{\partial}{\partial r}\left(r \frac{\partial v}{\partial r}\right)-\frac{v}{r^{2}}\right] \\
& \frac{\partial w}{\partial t}+u \frac{\partial w}{\partial x}+v \frac{\partial w}{\partial r}+\frac{v w}{r} \\
&=v\left[\frac{\partial^{2} w}{\partial r^{2}}+\frac{1}{r} \frac{\partial w}{\partial r}-\frac{w}{r^{2}}+\frac{\partial^{2} w}{\partial x^{2}}\right]-\frac{\sigma B_{0}^{2}}{\rho} w .
\end{aligned}
$$

Subject to the boundary conditions

$$
\begin{aligned}
& u(x, R)=0, \\
& v(x, R)=-V=-V_{0} \dot{R}, \\
& w(x, R)=\omega R, \\
& \left.\frac{\partial u}{\partial r}\right|_{r=0}=0, \\
& v(x, 0)=0, \\
& w(x, 0)=0,
\end{aligned}
$$

where $\rho$ is the density, $p$ is the pressure, $v$ is the kinematic viscosity, $B_{0}$ is the magnetic field, $V_{0}=V / \dot{R}$ is the measure of wall permeability $\left(V_{0}>0\right.$ corresponds to the injection and $V_{0}<0$ to suction), and $u, v$, and $w$ are the velocity components in $x$-, $r$-, and $\theta$-directions, respectively.

To normalize the governing equations (1)-(2) the following dimensionless quantities are used:

$$
\begin{aligned}
& \psi=v x f(\eta), \\
& \eta=\frac{r}{R}, \\
& p=\frac{\rho v^{2}}{R^{2}} P, \\
& u=\frac{v x}{R^{2} \eta} f^{\prime}, \\
& v=-\frac{v}{R \eta} f, \\
& w=\omega R g .
\end{aligned}
$$

Substituting (3) into (1)-(2), we get the following set of nonlinear differential equations:

$$
\begin{aligned}
& \frac{1}{\eta} \frac{d}{d \eta}\left(\eta \frac{d}{d \eta}\left(\frac{1}{\eta} f^{\prime}\right)\right)+\alpha\left(f^{\prime \prime}+\frac{f^{\prime}}{\eta}\right)-\frac{M^{2}}{\eta} f^{\prime} \\
& -\frac{1}{\eta^{2}}\left(\left(f^{\prime}\right)^{2}-f f^{\prime \prime}+\frac{f f^{\prime}}{\eta}\right)=\frac{1}{\xi} \frac{\partial P}{\partial \xi}, \\
& -\frac{d}{d \eta}\left(\frac{1}{\eta} f^{\prime}\right)-\alpha f^{\prime}+\frac{1}{\eta^{3}} f^{2}-\frac{1}{\eta^{2}} f f^{\prime}+\operatorname{Re}_{\omega}^{2} g=\frac{d P}{d \eta}, \\
& \frac{d}{d \eta}\left(\frac{1}{\eta} \frac{d}{d \eta}(\eta g)\right)-\left(\Omega \operatorname{Re}_{\omega}+M^{2}\right) g-\alpha\left(g-\eta g^{\prime}\right) \\
& \quad+\frac{1}{\eta} f g^{\prime}+\frac{1}{\eta^{2}} f g=0 .
\end{aligned}
$$


Subject to the boundary conditions

$$
\begin{aligned}
\lim _{\eta \rightarrow 0} \frac{d}{d \eta}\left(\frac{1}{\eta} f^{\prime}\right) & =0, \\
f(0) & =0, \\
g(0) & =0, \\
f^{\prime}(1) & =0, \\
f(1) & =\alpha V_{0}=\operatorname{Re}, \\
g(1) & =1,
\end{aligned}
$$

where prime denotes the differentiation with respect to $\eta$, $\alpha=R \dot{R} / \nu$ is the wall expansion parameter, such that its positive values indicate the tube contraction and negative values indicate expansion, $M^{2}=\sigma B_{0}^{2} R^{2} / \mu$ is the Hartmann number, $\operatorname{Re}_{\omega}=\omega R^{2} / \nu$ is the rotation Reynolds number, $\operatorname{Re}=R V / \nu$ is the injection Reynolds number, $\Omega=\dot{\omega} / \omega^{2}$ is the rotation parameter, and $\xi=x / R$ is the dimensionless axial coordinate.

Equations (4) and (5) can be further simplified by cross differentiation in order to obtain a fourth-order nonlinear differential equation given by

$$
\begin{aligned}
\frac{d}{d \eta}( & \left.\frac{1}{\eta} \frac{d}{d \eta}\left(\eta \frac{d}{d \eta}\left(\frac{1}{\eta} f^{\prime}\right)\right)\right) \\
= & \frac{f^{\prime}}{\eta^{2}}\left(f^{\prime \prime}-\frac{f^{\prime}}{\eta}\right)-\alpha\left(f^{\prime \prime \prime}+\frac{f^{\prime \prime}}{\eta}-\frac{f^{\prime}}{\eta^{2}}\right) \\
& -\frac{f}{\eta^{2}}\left(f^{\prime \prime \prime}-\frac{3 f^{\prime \prime}}{\eta}+\frac{3 f^{\prime}}{\eta^{2}}\right)+M^{2}\left(\frac{f^{\prime \prime}}{\eta}-\frac{f^{\prime}}{\eta^{2}}\right) .
\end{aligned}
$$
forms

The axial and tangential shear stresses $\tau_{r z}$ and $\tau_{r \theta}$ have the

$$
\begin{aligned}
& \tau_{x r}=\left.\mu \frac{\partial u}{\partial r}\right|_{r=R}=\frac{\mu \nu \xi}{R^{2}} f^{\prime \prime}(1), \\
& \tau_{\theta r}=\mu\left(\frac{\partial w}{\partial r}-\left.\frac{w}{r}\right|_{r=R}=\mu \omega R g^{\prime}(1)-\mu \omega .\right.
\end{aligned}
$$

The total shear stress $\tau_{w}$ at the surface of tube is

$$
\tau_{w}=\sqrt{\tau_{x r}^{2}+\tau_{\theta r}^{2}}
$$

and the skin friction coefficient is

$$
C_{f}=\frac{\tau_{w}}{\rho(\omega R)^{2}}=\frac{\xi}{\operatorname{Re}_{\omega}^{2}} \sqrt{\left(f^{\prime \prime}(1)\right)^{2}+\frac{\operatorname{Re}_{\omega}^{2}}{\xi^{2}}\left(g^{\prime}(1)-1\right)^{2}} .
$$

\section{Solution of the Problem}

In order to obtain the solution of the nonlinear ordinary differential equations (6) and (8) along with the boundary conditions (7) the homotopy analysis method (HAM) is used for which the following initial guesses are chosen:

$$
\begin{aligned}
& f_{0}(\eta)=\alpha V_{0}\left(2-\eta^{2}\right) \eta^{2}, \\
& g_{0}(\eta)=\eta,
\end{aligned}
$$

and the auxiliary linear operators are

$$
\begin{aligned}
\mathscr{L}_{1} & \equiv \frac{d}{d \eta}\left(\frac{1}{\eta} \frac{d}{d \eta}(\eta H)\right), \\
\mathscr{L}_{2} g & =H(\eta g), \\
H & \equiv \frac{d}{d \eta}\left(\frac{1}{\eta} \frac{d}{d \eta}\right) .
\end{aligned}
$$

Since the HAM is well known and is broadly used by the community of fluid dynamics, therefore it seems appropriate to conceal the procedural details of the method. However, the readers are referred to Liao [18] for details about procedure of the HAM solution. We here directly write the $m$ th-order deformation equation (for $m \geq 1$ ) given by

$$
\begin{aligned}
& \mathscr{L}_{1}\left[f_{m}(\eta)-\chi_{m} f_{m-1}(\eta)\right]=\hbar_{1} \mathscr{R}_{1 m}(\eta), \\
& \mathscr{L}_{2}\left[g_{m}(\eta)-\chi_{m} g_{m-1}(\eta)\right]=\hbar_{2} \mathscr{R}_{2 m}(\eta) .
\end{aligned}
$$

Subject to the boundary conditions

$$
\begin{aligned}
f_{m}(0) & =\lim _{\eta \rightarrow 0} H\left(f_{m}\right)=g_{m}(0)=f_{m}(1)=f_{m}^{\prime}(1) \\
& =g_{m}(1)=0,
\end{aligned}
$$

where

$$
\begin{aligned}
& \mathscr{R}_{1 m}(\eta)= H\left(\eta H\left(f_{m-1}\right)\right) \\
&+\alpha\left(f_{m-1}^{\prime \prime \prime}+\frac{f_{m-1}^{\prime \prime}}{\eta}-\frac{f_{m-1}^{\prime}}{\eta^{2}}\right) \\
&-\frac{M^{2}}{\eta}\left(f_{m-1}^{\prime \prime}-\frac{f_{m-1}^{\prime}}{\eta}\right) \\
&+\frac{1}{\eta^{2}} \sum_{k=0}^{m-1} f_{m-1-k}\left(f_{k}^{\prime \prime \prime}-\frac{3 f_{k}^{\prime \prime}}{\eta}+\frac{3 f_{k}^{\prime}}{\eta^{2}}\right) \\
&-\frac{1}{\eta^{2}} \sum_{k=0}^{m-1} f_{m-1-k}^{\prime}\left(f_{k}^{\prime \prime}-\frac{f_{k}^{\prime}}{\eta}\right), \\
& \mathscr{R}_{2 m}(\eta)= H\left(\eta g_{m-1}\right)-\left(\Omega \mathrm{Re}_{\omega}+M^{2}\right) g_{m-1} \\
&+\frac{\alpha}{\eta} \sum_{k=0}^{m-1} f_{m-1-k}\left(g_{k}^{\prime}+\frac{1}{\eta} g_{k}\right), \\
& \chi_{m}=\left\{\begin{array}{l}
0, \\
\text { for } m \leq 1, \\
1,
\end{array} \text { for } m>1 .\right.
\end{aligned}
$$

For a particular value of " $m$ ", (14) are ordinary differential equations which can easily be solved by a standard method. 
The final solution can be written in the form of an infinite series of functions:

$$
\begin{aligned}
& f(\eta)=f_{0}(\eta)+\sum_{m=1}^{\infty} f_{m}(\eta), \\
& g(\eta)=g_{0}(\eta)+\sum_{m=1}^{\infty} g_{m}(\eta),
\end{aligned}
$$

where $f_{m}(\eta)$ and $g_{m}(\eta)$ are the solutions of $m$ th $(m \geq 1)$ order deformation equations.

3.1. Convergence of the Series Solution. In order to verify that the series (17) is the approximate solutions of the differential equations (6) and (8) along with boundary conditions (7) it is necessary to show the convergence of the solution series. As mentioned by Liao [18] the convergence of the solution series strongly depends upon the auxiliary parameters $\hbar_{1}$ and $\hbar_{2}$ once the initial guesses and the linear operators have been selected. A rough estimate for the permissible regions of the values of $\hbar_{1}$ and $\hbar_{2}$ can be obtained by plotting the so-called $\hbar$-curves. In Figure 2 the $\hbar$-curves are plotted by keeping the other parameters fixed. From the figure it can be seen that the interval of allowed values of $\hbar_{1}$ and $\hbar_{2}$ is between -1.7 and -0.2 (roughly). However, to obtain the optimal values of $\hbar_{1}$ and $\hbar_{2}$ it is convenient to calculate the squared residual error. In Table 1 we have calculated the squared residual error at different orders of approximation for fixed values of the other parameters. From the table it is clear that as the order of approximation increases the squared residual errors decrease and are confined in an admissible range. The corresponding optimal values for $\hbar_{1}$ and $\hbar_{2}$ can be obtained by minimizing the squared residual error using the command "NMinimize" of the software Mathematica. In Table 1 we also calculate the HAM solution at different orders and corresponding optimal values of $\hbar_{1}$ and $\hbar_{2}$. Clearly, there are no corrections after 10th order of approximation up to 8 decimal places. This proves the convergence and accuracy of our series solution. A comparison of the current analytic solution is given with the numerical and asymptotic solution reported by Li et al. [17] in Table 2. The table shows a good agreement up to 4 decimal places between our results and the numerical results provided in $\mathrm{Li}$ et al. [17].

\section{Flow Characteristics}

In this section we analyse the flow characteristics of the problem by plotting the graphs of velocity profile, wall skin friction, and the pressure variations in the tube for various values of the parameters. The axial velocity profile versus $\eta$ for various values of wall expansion parameter $\alpha$ can be seen in Figure 3. From the figure it is clear that as contraction increases the velocity profile also increases. It is further observed that the fluid velocity decreases as the tube wall shifts from contraction to expansion state. It can be noticed that as the tube either expands or contracts, the magnitude of velocity increases. However, for increasing expansion rate the backward flow accelerates, whereas, for increasing contraction, the forward flow exaggerates. Figure 4

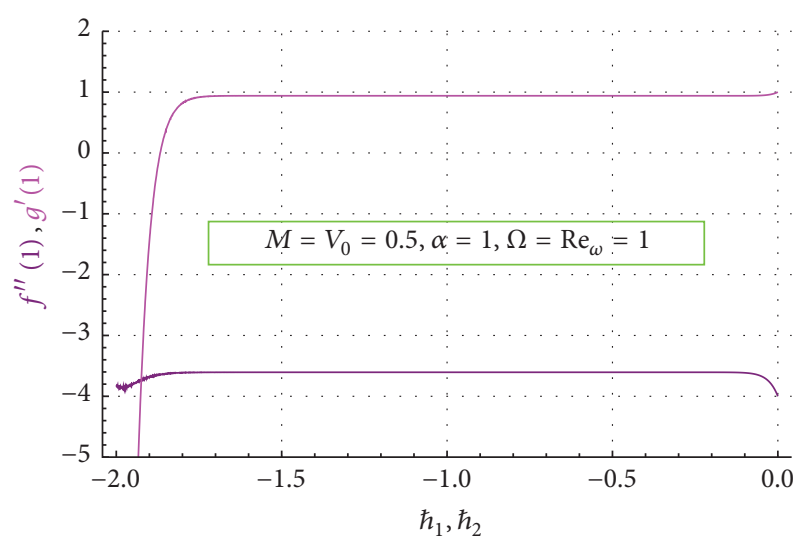

FIGURE 2 : $\hbar$-curves at 30 th order of approximation.

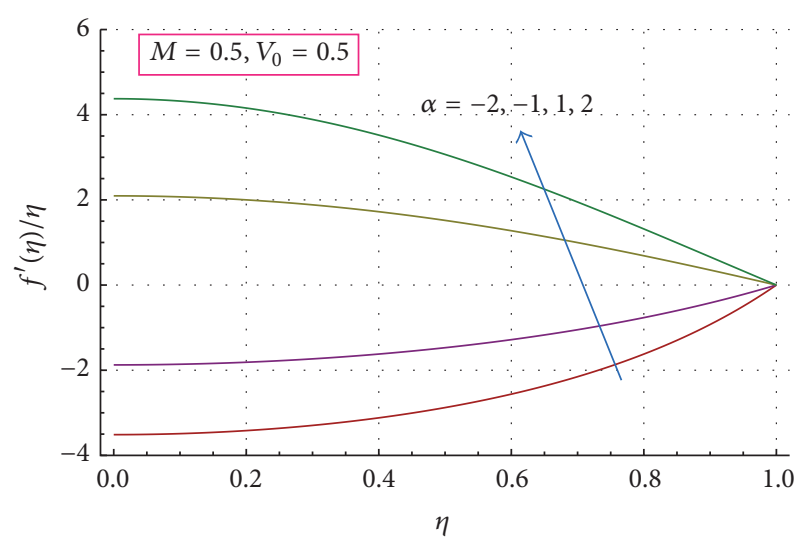

FIGURE 3: Effect of wall expansion parameter $\alpha$ on the axial velocity.

has been prepared to express the variation in the tangential velocity of fluid induced by the expansion and contraction of tube wall. This figure shows that for increasing expansion rate the tangential velocity profile decreases, while for increasing the contraction rate the tangential velocity increases.

Figures 5 and 6 are plotted to discuss the effects of the Hartmann number $M$ on the axial and tangential velocity profiles, respectively. Figure 5 indicates that the axial velocity is large in case of hydrodynamic flow as compared to the magnetohydrodynamic flow near the tube centre. However, a reverse behaviour can be seen near the tube boundary. Here axial velocity is going to be augmented when large values of $M$ are taken into contemplation. Figure 6 reveals that the tangential velocity of fluid improves by increasing the values of $M$ throughout the tube. In this case magnetic field acts as an opposing force which resists the tangential velocity.

Figure 7 shows the combined effects of wall expansion/contraction parameter $\alpha$ and the Hartmann number $M$ on the wall skin friction coefficient. A close look at this Figure informs that, for the state of tube expansion, the magnetic field works as a supporting force to enhance the skin friction coefficient, while, for tube contraction state, the magnetic field becomes a resistive force. Whether the tube is in expansion state or in contraction state, the magnitude of skin friction always increases as $M$ increases. The value $\alpha=0$ 
TABLE 1: Convergence and accuracy of HAM solution at different orders of approximation when $\alpha=1, M=0.5, V_{0}=0.5, \Omega=1, \xi=1$, and $N=20$.

\begin{tabular}{|c|c|c|c|c|c|c|}
\hline $\begin{array}{l}\text { Order of } \\
\text { approximation } m\end{array}$ & $f^{\prime \prime}(1)$ & $g^{\prime}(1)$ & $\begin{array}{l}\text { Residual error } E_{1 m} \\
\text { for (8) }\end{array}$ & $\begin{array}{l}\text { Residual error } E_{2 m} \\
\text { for }(6)\end{array}$ & Optimal $\hbar_{1}$ & Optimal $\hbar_{2}$ \\
\hline 2 & -3.60185569 & 0.98963266 & $5.756 \times 10^{-2}$ & $3.832 \times 10^{-3}$ & -0.897 & -0.916 \\
\hline 5 & -3.60529192 & 0.98947505 & $1.006 \times 10^{-4}$ & $3.256 \times 10^{-6}$ & -0.948 & -0.992 \\
\hline 10 & -3.60529631 & 0.98947481 & $1.774 \times 10^{-9}$ & $4.968 \times 10^{-11}$ & -0.932 & -0.997 \\
\hline 15 & -3.60529631 & 0.98947481 & $3.615 \times 10^{-11}$ & $1.024 \times 10^{-12}$ & -0.845 & -0.832 \\
\hline 20 & -3.60529631 & 0.98947481 & $3.561 \times 10^{-10}$ & $4.223 \times 10^{-11}$ & -0.689 & -0.865 \\
\hline 25 & -3.60529631 & 0.98947481 & $1.808 \times 10^{-9}$ & $1.753 \times 10^{-11}$ & -0.580 & -0.621 \\
\hline 30 & -3.60529631 & 0.98947481 & $3.981 \times 10^{-9}$ & $3.781 \times 10^{-11}$ & -0.478 & -0.518 \\
\hline
\end{tabular}

TABLE 2: Comparison of current analytic solution by HAM with the solution reported by Li et al. [17] for $f^{\prime \prime}(1) / 4 \operatorname{Re}$ at $\alpha=0.05$.

\begin{tabular}{lccc}
\hline Re & $\begin{array}{c}\text { Asymptotic } \\
\text { solution by Li et al. } \\
{[17]}\end{array}$ & $\begin{array}{c}\text { Numerical solution } \\
\text { by Li et al. [17] }\end{array}$ & $\begin{array}{c}\text { Current HAM } \\
\text { solution }\end{array}$ \\
\hline 0.592 & -2.0841 & -2.0694 & -2.0694 \\
0.297 & -2.0339 & -2.0299 & -2.0299 \\
0.041 & -1.9903 & -1.9903 & -1.9903 \\
-0.052 & 1.9745 & 1.9744 & 1.9744 \\
-0.141 & 1.9593 & 1.9584 & 1.9584 \\
-0.422 & 1.9115 & 1.9015 & 1.9015 \\
\hline
\end{tabular}

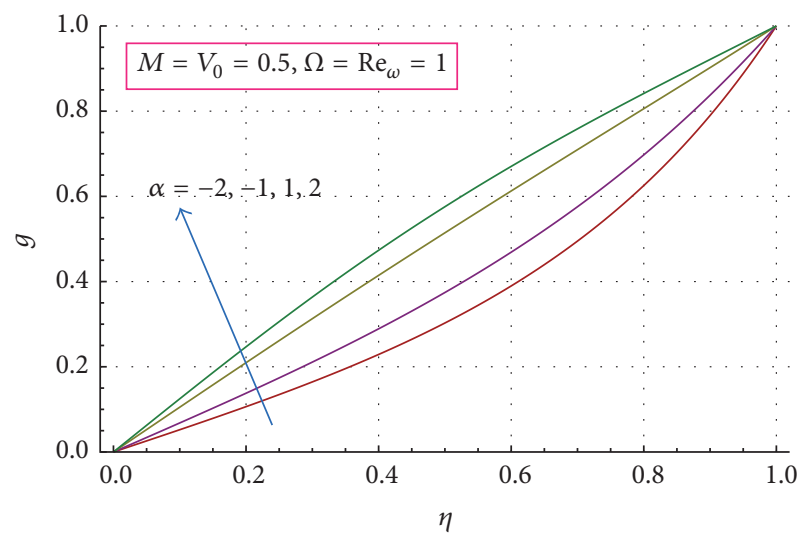

FIGURE 4: Effect of wall expansion parameter $\alpha$ on the tangential velocity.

corresponds to the rest state (when the tube neither contracts nor expands). In the rest state of tube, the value of skin friction is zero which indicates no flow across the tube. The influence of permeability parameter $V_{0}$ on the axial and tangential velocities is presented by Figures 8 and 9 . These figures are portrayed when the tube is observed to be in contracting state $(\alpha=2)$. Figure 8 renders that the axial velocity of fluid improves as one moves from the suction regime to the injection. Moreover, the variations in velocity are more noticeable near the tube centre. Figure 9 demonstrates that the tangential velocity of the fluid flowing

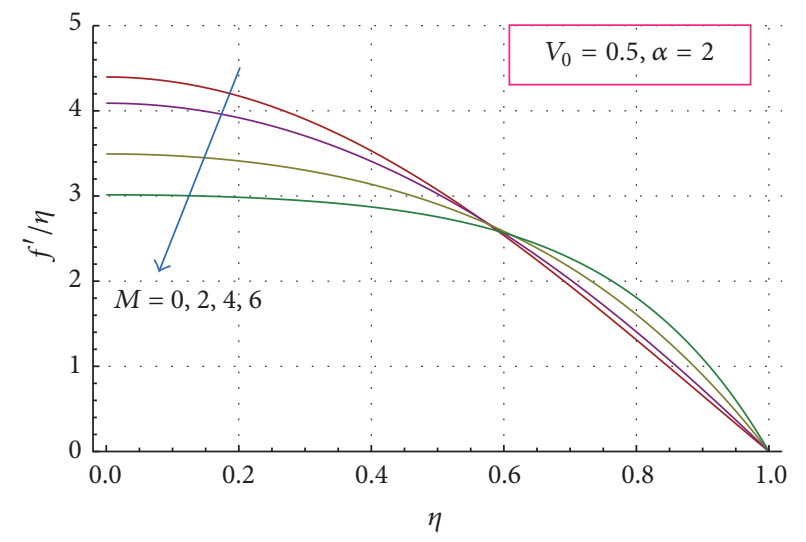

FIGURE 5: Effect of the Hartmann number $M$ on the axial velocity.

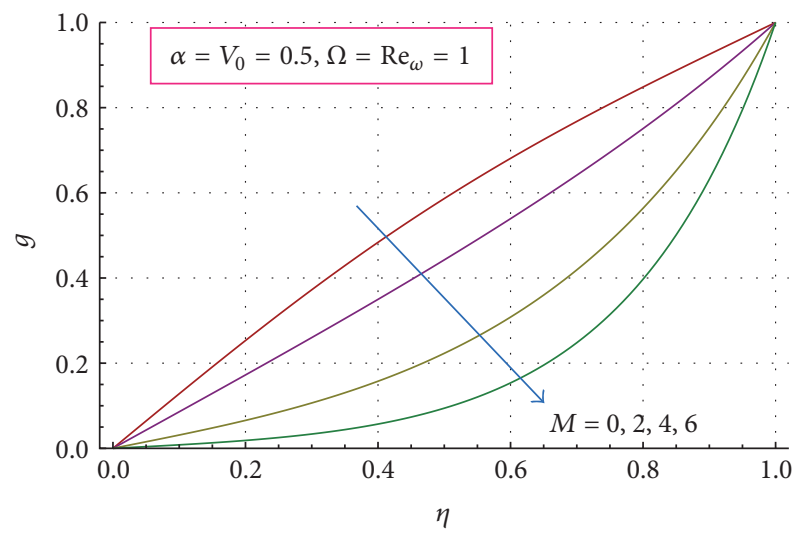

Figure 6: Effect of the Hartmann number $M$ on the tangential velocity.

through the tube increases by increasing injection and of course the tangential velocity profile decreases in the case when the suction phenomenon becomes strong.

The skin friction coefficient for various values of permeability parameter $V_{0}$ against $\alpha$ has been plotted in Figure 10 . Clearly, two different variation trends are noticed for various values of $V_{0}$. From the figure it is clear that for the case of tube contraction the skin friction becomes a decreasing function of $V_{0}$, whereas the skin friction coefficient is going to be 


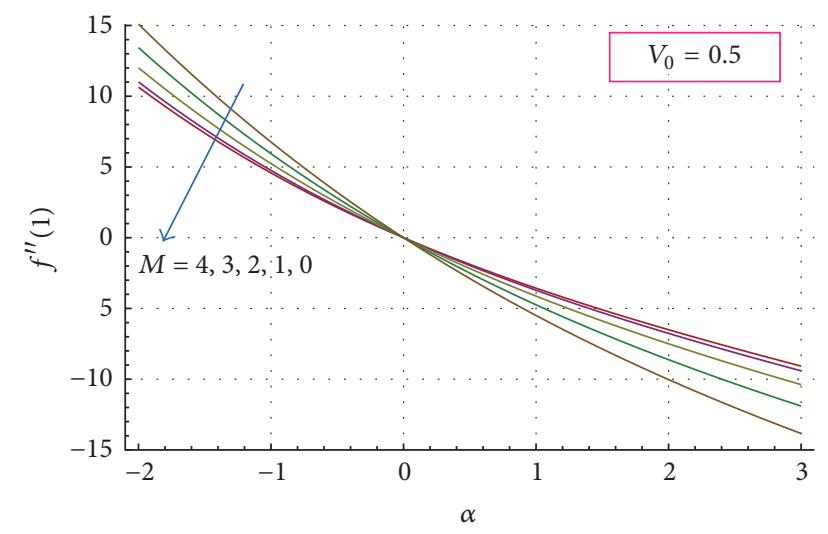

FIGURE 7: The skin friction coefficient against $\alpha$ for various values of M.

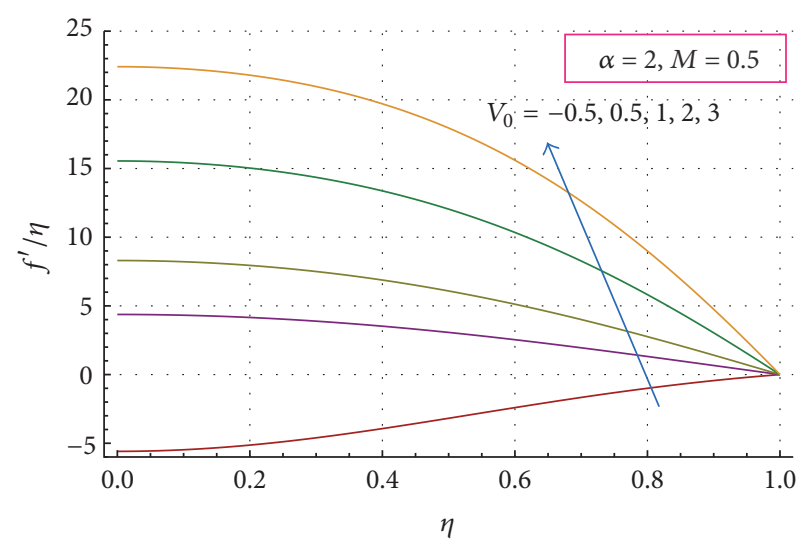

FIGURE 8: Effect of the suction/injection parameter $V_{0}$ on the axial velocity.

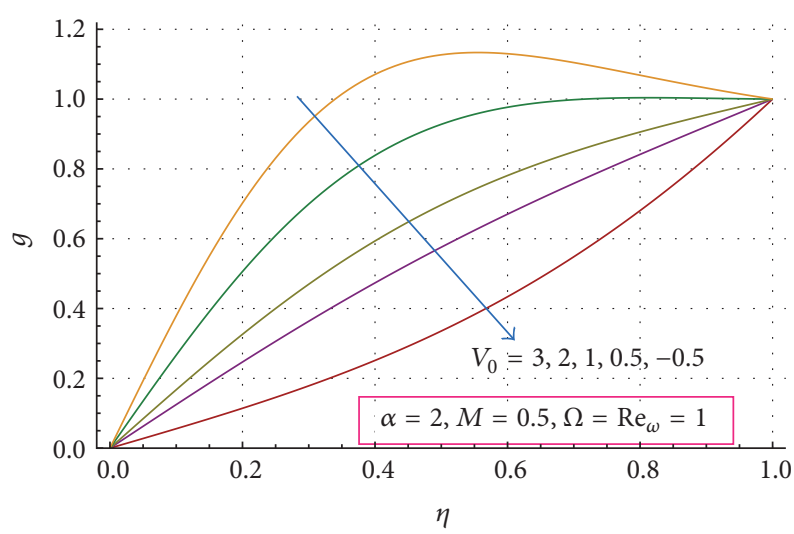

FIGURE 9: Effect of the parameter $V_{0}$ on the tangential velocity.

enhanced by increasing $V_{0}$ in expansion state. This means that when tube contracts the presence of strong injection strengthens the negative skin friction or in other words, injection causes the forward flow to be enhanced when cylinder contracts. The consequence of rotational Reynolds number $\mathrm{Re}_{\omega}$ on the tangential velocity has been captured in Figure 11 when the tube is in contracting state. This figure

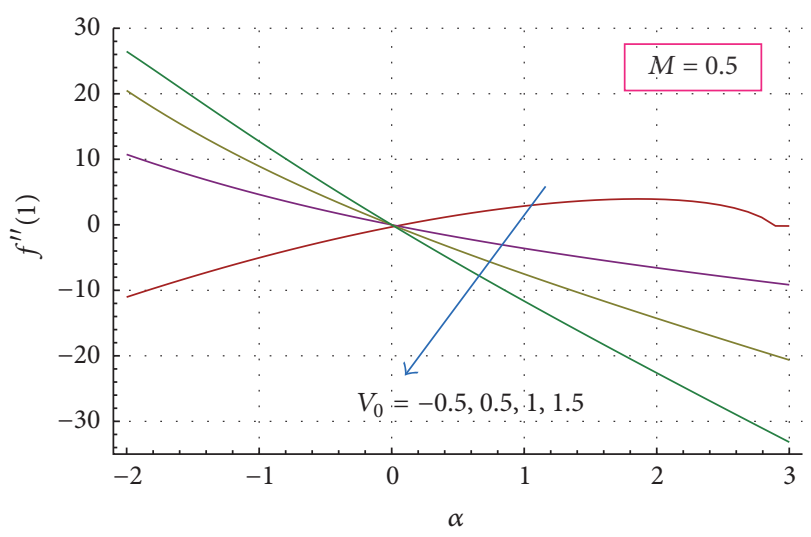

FIGURE 10: The skin friction coefficient against $\alpha$ for various values of $V_{0}$.

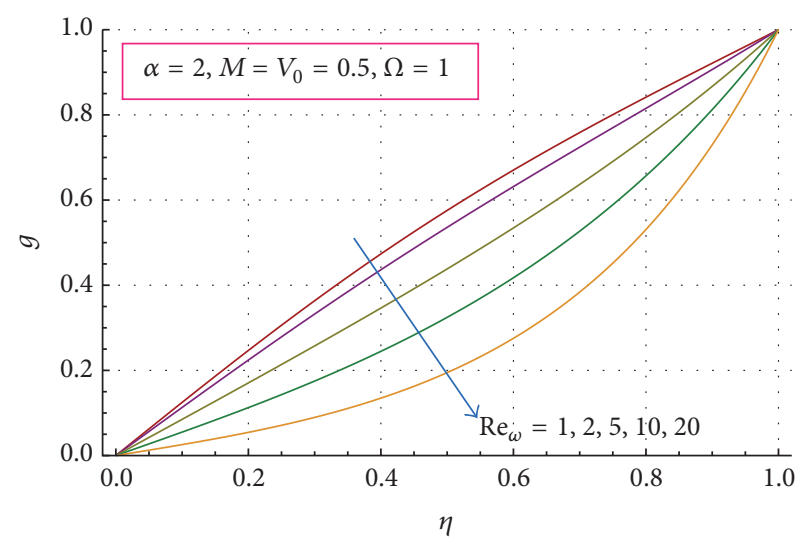

FIGURE 11: Effect of the rotational Reynolds number $\operatorname{Re}_{\omega}$ on the tangential velocity.

depicts that the fluid tangential velocity decreases when large values of $\mathrm{Re}_{\omega}$ are taken into account. This conduct of fluid velocity is of course due to the presence of large inertial forces which resist the fluid tangential velocity.

The variations in the pressure versus $\eta$ for different emerging parameters have been shown through Figures 1215. In this regard, the selected parameters of concern are $\alpha$, $M, V_{0}$, and $\operatorname{Re}_{\omega}$. Figure 12 notifies that the pressure gradient increases and becomes adverse near the tube walls when tube is going to be relaxed back from the expansion state. Also in different phases of expansion and contraction states the pressure gradient shows miscellaneous variations throughout the tube. A careful glance at this figure reveals that the pressure variation curve becomes more concave when tube is going to be contracted more. At $\alpha=2$ it is noticed that the pressure gradient becomes favourable near the centre of tube while near the wall it becomes more adverse. In Figure 13, it is observed that the pressure gradient becomes more favourable for large values of $M$ in the region $0 \leq \eta<0.86$ (roughly), although, in the region $0.86<\eta \leq 1$, the magnetic force accelerates the adverse pressure gradient. Figure 14 reports that the variation in the values of permeability parameter $V_{0}$ has miscellaneous effects on the pressure gradient. In this 


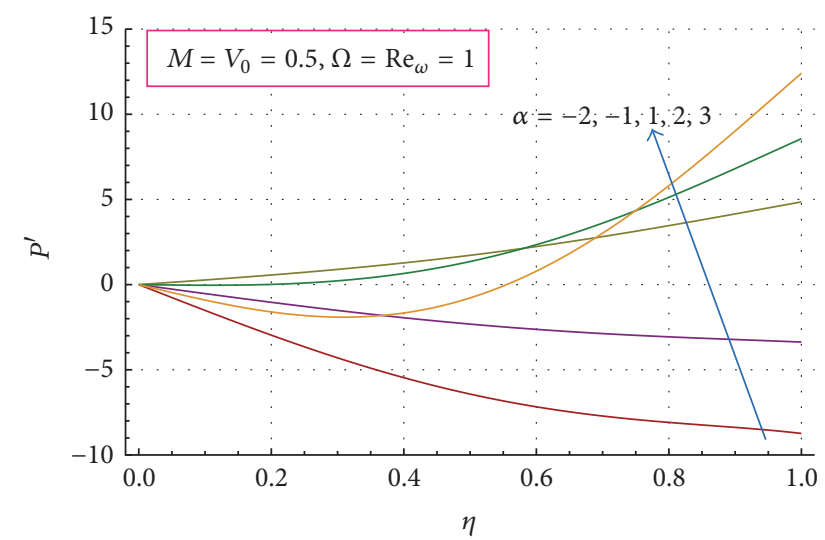

Figure 12: Pressure variation in the tube for different values of the parameter $\alpha$.

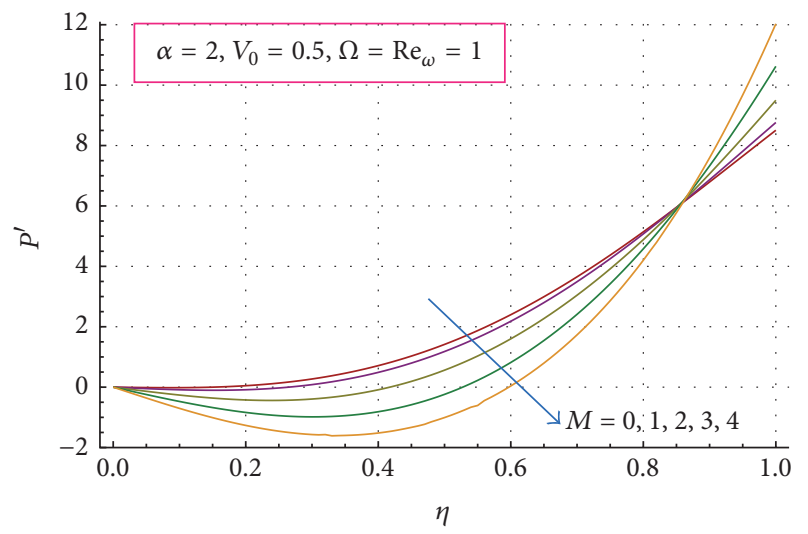

FIGURE 13: Pressure variation in the tube for different values of the Hartmann number $M$.

figure, it is noted that the curve for the pressure gradient changes from linear to parabolic shape as we alter from suction to injection situation. For the case of remarkable injection effects, the favourable pressure gradient increases near the tube centre when compared to its behaviour near the tube wall. Figure 15 explains that the adverse pressure gradient increases for large values of $\mathrm{Re}_{\omega}$ throughout the tube. The variation in pressure accumulates near the tube centre and becomes more noticeable near the tube boundary.

\section{Conclusion}

The magnetohydrodynamic rotating flow inside a uniformly porous tube with contracting/expanding wall is investigated. The analysis is based on the analytic technique HAM with the help of which we obtained an approximate series solution. The solution series is uniformly valid for all range of the involved parameters. The physical results categorically indicate the following findings:

(i) The magnitude of skin friction increases as the expansion/contraction rates increase.

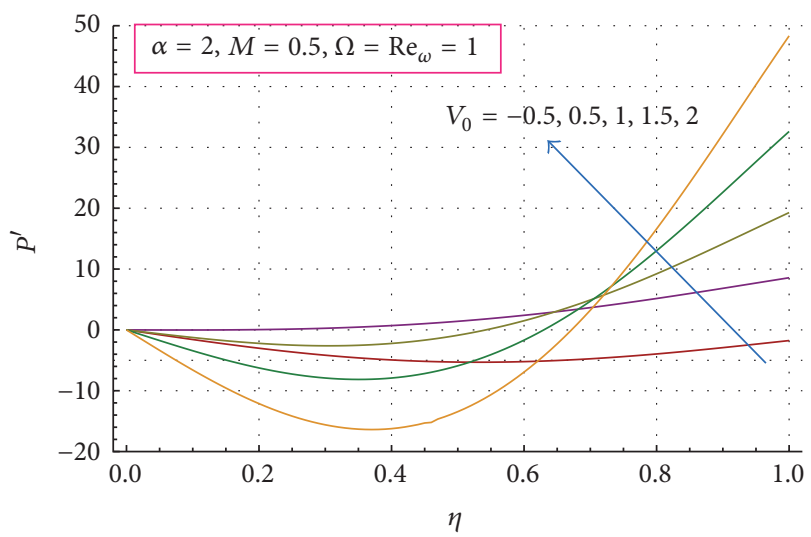

FIGURE 14: Effect of the suction/injection parameter $V_{0}$ on pressure variation in the tube.

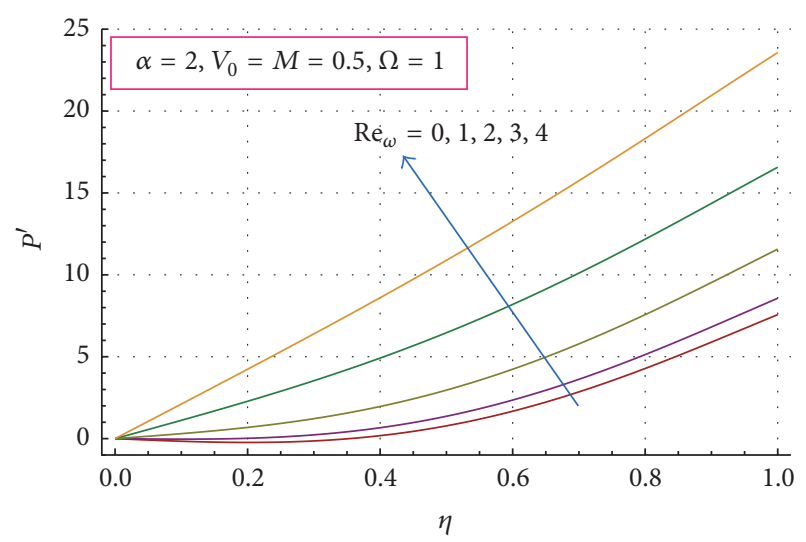

FIGURE 15: Effect of the rotation Reynolds number $\mathrm{Re}_{\omega}$ on pressure variation in the tube.

(ii) The contraction of tube accelerates the torsional velocity of fluid particles while expansion of tube depreciates the rotating velocity of fluid.

(iii) The axial velocity has two variation trends as magnetic field intensifies; near the centre, the velocity is a decreasing function of the Hartmann number and near the wall the velocity is an increasing function of magnetic field.

(iv) Also the magnitude of skin friction increases for both expansion and contraction as the magnetic field becomes stronger.

(v) The pressure variation is large near the wall of pipe and vanishes near the centre of pipe.

(vi) The large contraction rate results in the favourable pressure gradient near the centre of the tube while at the wall adverse pressure gradient magnifies.

(vii) The augmentation of magnetic field results in the increasing of favourable pressure gradient in tube centre while it increases the adverse pressure gradient near the wall. 
(viii) An increase in the Reynolds number $\mathrm{Re}_{\omega}$ is to increase the adverse pressure gradient.

\section{Nomenclature}

Latin Symbols

$\begin{array}{ll}B_{0}: & \text { Magnetic field } \\ C_{f}: & \text { Skin friction coefficient } \\ f_{m}: & \text { mth-order HAM solution } \\ f: & \text { Dimensionless stream function } \\ g: & \text { Dimensionless rotation velocity } \\ M: & \text { The Hartmann number } \\ p: & \text { Pressure } \\ P: & \text { Dimensionless pressure } \\ R: & \text { Radius of tube } \\ \operatorname{Re}_{0} & \text { Injection Reynolds number } \\ \operatorname{Re}_{\omega}: & \text { Rotation Reynolds number } \\ t: & \text { Dimensional time } \\ (u, v, w): \text { Velocity components in }(x, r, \theta) \text { directions } \\ V: & \text { Wall permeability } \\ V_{0}: & \text { Dimensionless wall permeability } \\ (x, r, \theta): & \text { Axial, radial, and azimuthal coordinates, } \\ & \text { respectively. }\end{array}$

Greek Symbols

$\begin{array}{ll}\alpha: & \text { Wall expansion parameter } \\ \nu: & \text { Kinematic viscosity } \\ \rho: & \text { Density of fluid } \\ \psi: & \text { Stream function } \\ \sigma: & \text { Electrical conductivity } \\ \eta: & \text { Dimensionless radial axis } \\ \omega: & \text { Angular velocity } \\ \Omega: & \text { Rotation parameter } \\ \mu: & \text { Dynamic viscosity } \\ \xi: & \text { Dimensionless axial axis } \\ \tau_{w}: & \text { Shear stress } \\ \hbar_{1}, \hbar_{2}: & \text { Convergence controlling parameters. }\end{array}$

\section{Competing Interests}

The author declares that there is no conflict of interests regarding the publication of this manuscript.

\section{References}

[1] S. Uchida and H. Aoki, "Unsteady flows in a semi-infinite contracting or expanding pipe," Journal of Fluid Mechanics, vol. 82, no. 2, pp. 371-387, 1977.

[2] M. Ohki, "Unsteady flow in a porous, elastic, circular tubeI. The wall contracting or expanding in an axial direction," Bulletin of the JSME, vol. 23, no. 179, pp. 679-686, 1980.

[3] M. Goto and S. Uchida, "Unsteady flows in a semi-infinite expanding pipe with injection through wall," Transactions of the Japan Society for Aeronautical and Space Sciences, vol. 38, no. 434, pp. 131-138, 1990 .
[4] N. M. Bujurke, N. P. Pai, and G. Jayaraman, "Computer extended series solution for unsteady flow in a contracting or expanding pipe," IMA Journal of Applied Mathematics, vol. 60, no. 2, pp. 151-165, 1998.

[5] X. Si, L. Zheng, X. Zhang, M. Li, J. Yang, and Y. Chao, "Multiple solutions for the laminar flow in a porous pipe with suction at slowly expanding or contracting wall," Applied Mathematics and Computation, vol. 218, no. 7, pp. 3515-3521, 2011.

[6] S. Srinivas, A. S. Reddy, and T. R. Ramamohan, "A study on thermal-diffusion and diffusion-thermo effects in a twodimensional viscous flow between slowly expanding or contracting walls with weak permeability," International Journal of Heat and Mass Transfer, vol. 55, no. 11-12, pp. 3008-3020, 2012.

[7] S. Srinivas, A. S. Reddy, and T. R. Ramamohan, "Mass transfer effects on viscous flow in an expanding or contracting porous pipe with chemical reaction," Heat Transfer-Asian Research, vol. 44, no. 6, pp. 552-567, 2015.

[8] S. Srinivas, A. Subramanyam Reddy, T. R. Ramamohan, and A. K. Shukla, "Influence of heat transfer on MHD flow in a pipe with expanding or contracting permeable wall," Ain Shams Engineering Journal, vol. 5, no. 3, pp. 817-830, 2014.

[9] R. D. O'Dea and S. L. Waters, "Flow and solute uptake in a twisting tube," Journal of Fluid Mechanics, vol. 562, pp. 173-182, 2006.

[10] O. D. Makinde, "Collapsible tube flow: a mathematical model," Romanian Journal of Physics, vol. 50, pp. 493-506, 2005.

[11] O. D. Makinde, "Heat and mass transfer in a pipe with moving surface: effects of viscosity variation and energy dissipation," Quaestiones Mathematicae. Journal of the South African Mathematical Society, vol. 24, no. 1, pp. 93-104, 2001.

[12] S. Xinhui, Z. Liancun, C. Xuehui, Z. Xinxin, C. Limei, and L. Min, "The effects of slip velocity on a micropolar fluid through a porous channel with expanding or contracting walls," Computer Methods in Biomechanics and Biomedical Engineering, vol. 17, no. 4, pp. 423-432, 2014.

[13] M. A. Sprague and P. D. Weidman, "Three-dimensional flow induced by the torsional motion of a cylinder," Fluid Dynamics Research, vol. 43, no. 1, Article ID 015501, 2011.

[14] S. Munawar, N. Saleem, and A. Mehmood, "Entropy production in the flow over a swirling stretchable cylinder," Thermophysics and Aeromechanics, vol. 23, no. 3, pp. 435-444, 2016.

[15] T. Fang and S. Yao, "Viscous swirling flow over a stretching cylinder," Chinese Physics Letters, vol. 28, no. 11, Article ID 114702, 2011.

[16] T.-G. Fang, J. Zhang, Y.-F. Zhong, and H. Tao, "Unsteady viscous flow over an expanding stretching cylinder," Chinese Physics Letters, vol. 28, no. 12, Article ID 124707, 2011.

[17] L. Li, P. Lin, X. Si, and L. Zheng, "A numerical study for multiple solutions of a singular boundary value problem arising from laminar flow in a porous pipe with moving wall," Journal of Computational and Applied Mathematics, vol. 313, pp. 536-549, 2017.

[18] S. J. Liao, Beyond Perturbation: Introduction to Homotopy Analysis Method, Chapman and Hall/CRC, London, UK, 2003. 


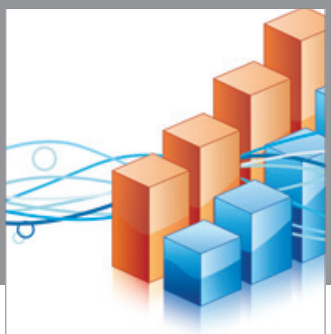

Advances in

Operations Research

vatem alat4

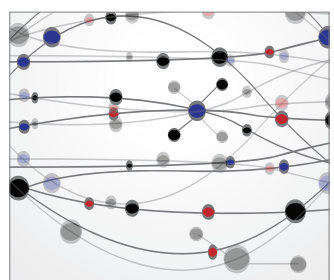

\section{The Scientific} World Journal
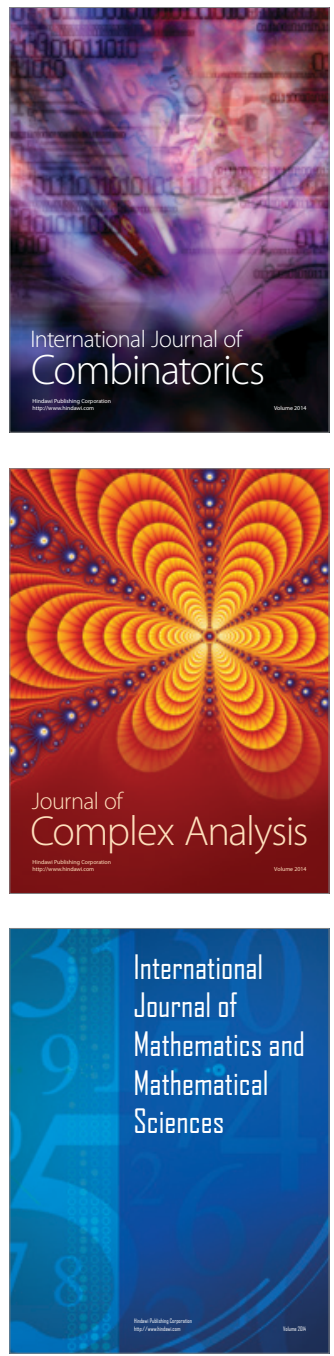
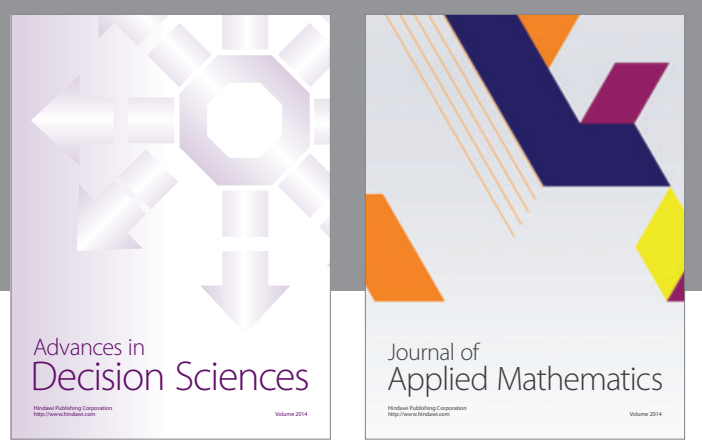

Algebra

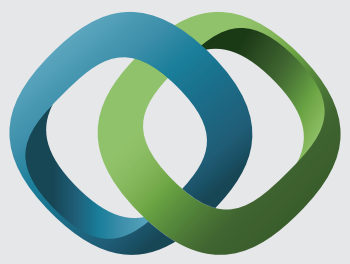

\section{Hindawi}

Submit your manuscripts at

https://www.hindawi.com
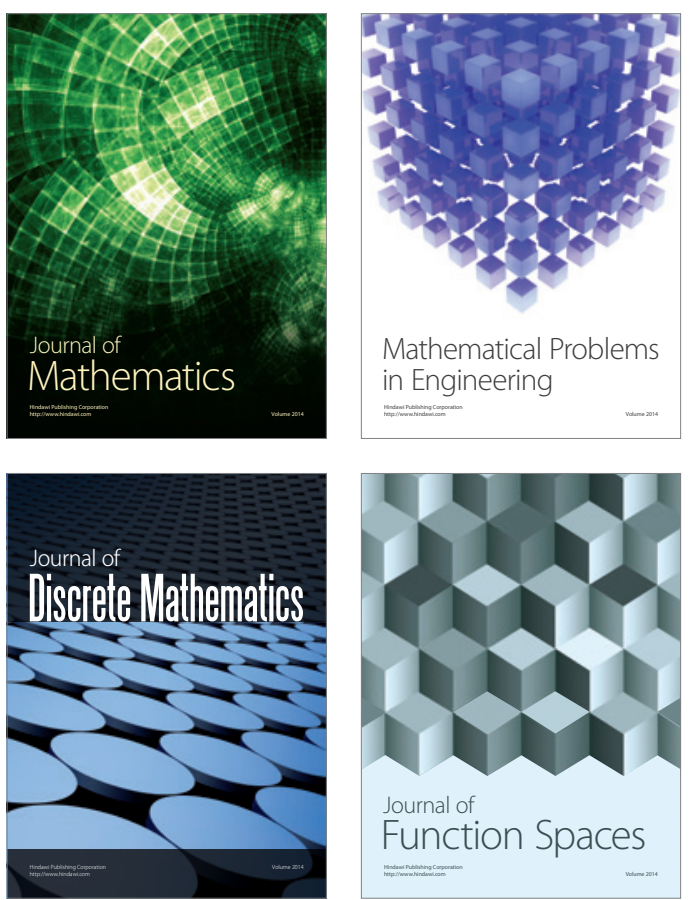

Mathematical Problems in Engineering
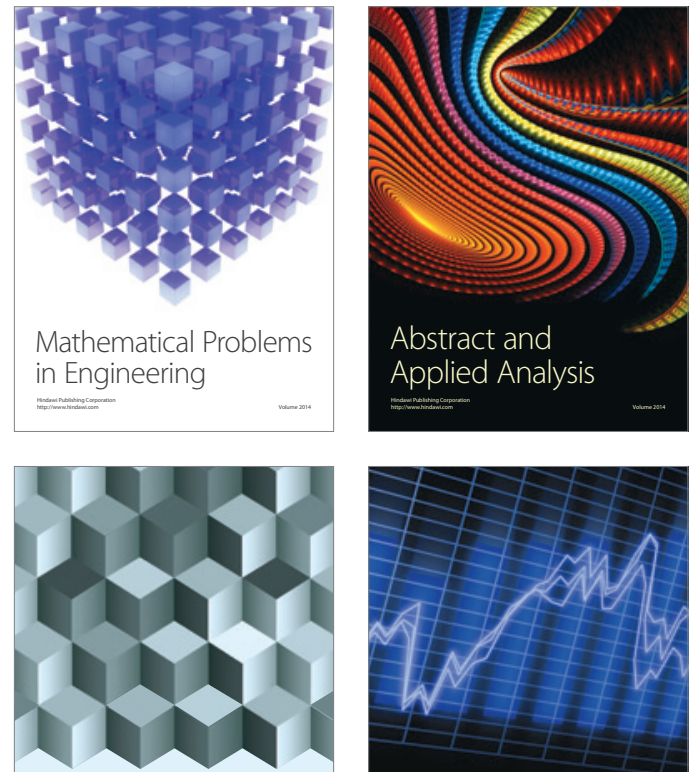

Journal of

Function Spaces

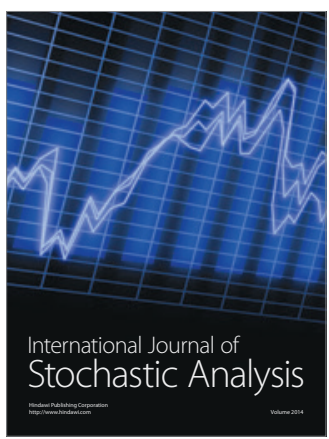

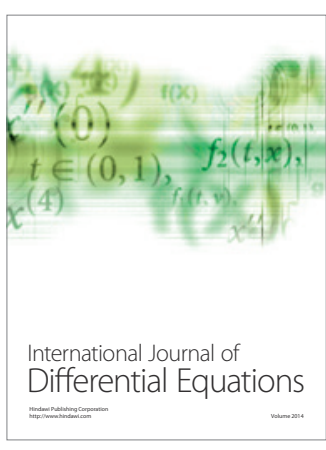
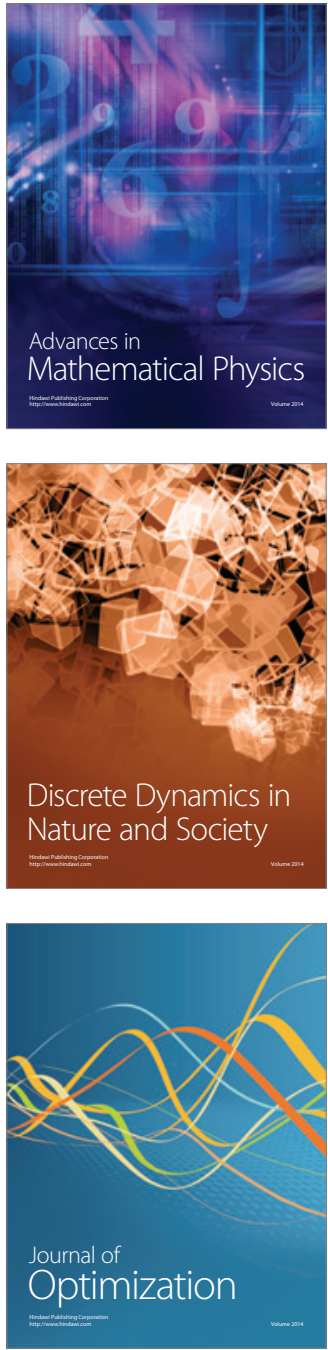